\title{
HIGHER-ORDER NONLINEAR DISPERSIVE EQUATIONS
}

\author{
CARLOS E. KENIG, GUSTAVO PONCE, AND LUIS VEGA
}

(Communicated by Barbara Lee Keyfitz)

ABSTRACT. We study nonlinear dispersive equations of the form

$$
\partial_{t} u+\partial_{x}^{2 j+1} u+P\left(u, \partial_{x} u, \ldots, \partial_{x}^{2 j} u\right)=0, \quad x, t \in \mathbb{R}, \quad j \in \mathbb{Z}^{+},
$$

where $P(\cdot)$ is a polynomial having no constant or linear terms. It is shown that the associated initial value problem is locally well posed in weighted Sobolev spaces. The method of proof combines several sharp estimates for solutions of the associated linear problem and a change of dependent variable which allows us to consider data of arbitrary size.

\section{INTRODUCTION}

In this paper we consider the initial value problem

$$
\left\{\begin{array}{l}
\partial_{t} u+\partial_{x}^{2 j+1} u+P\left(u, \partial_{x} u, \ldots, \partial_{x}^{2 j} u\right)=0, \quad x, t \in \mathbb{R}, j \in \mathbb{Z}^{+}, \\
u(x, 0)=u_{0}(x)
\end{array}\right.
$$

where $\partial_{t}=\partial / \partial t, \partial_{x}=\partial / \partial x, u=u(x, t)$ is a real- (or complex-) valued function, and

$$
P: \mathbb{R}^{2 j+1} \rightarrow \mathbb{R} \quad\left(\text { or } P: \mathbb{C}^{2 j+1} \rightarrow \mathbb{C}\right)
$$

is a polynomial having no constant or linear terms; i.e.,

$$
P(z)=\sum_{|\alpha|=\ell_{0}}^{\ell_{1}} a_{\alpha} z^{\alpha} \quad \text { with } \ell_{0} \geq 2
$$

and $z=\left(z_{1}, \ldots, z_{2 j+1}\right)$.

The class described in (1.1) generalizes several models arising in both mathematics and physics. In particular, it contains the KdV hierarchy [14], higherorder models in water waves problems and in elastic media (see [12] and references therein), and the equations discussed in [3, §7].

Our purpose is to study local well-posedness of the IVP (1.1). Here the difficulties appear from the fact that, in general, techniques such as standard energy estimates, space-time $\left(L_{x}^{p} L_{t}^{q}-\right)$ estimates, Galerkin's method, and so on cannot be applied.

Received by the editors October 19, 1992 and, in revised form, December 7, 1992.

1991 Mathematics Subject Classification. Primary 35Q30; Secondary 35G25, 35D99.

Key words and phrases. Higher order models, smoothing effects, gauge transformation.

The first and second authors were supported by an NSF grant.

The third author was supported by a DGICYT grant. 
In [10] we showed that (1.1) is locally well posed for small data $u_{0} \in H^{s}(\mathbb{R}) \cap$ $L^{2}\left(|x|^{m} d x\right) \equiv X_{s, m}$. There the smallness assumption on the data was removed for $P(\cdot)$ 's independent of the highest derivative $\partial_{x}^{2 j} u$ (for related results see $[2,17,18,19])$. Our main result here establishes the local well-posedness of the IVP (1.1) in $X_{s, m}$ without any size restriction on the data. To achieve it we include the fact that the results in [10] extend to diagonal systems and a change of dependent variable. This allows us to write the equation in (1.1) (after a few differentiations with respect to the $x$-variable) as a diagonal system

$$
\partial_{t} \omega^{k}+\partial_{x}^{2 j+1} \omega^{k}+Q_{k}\left(\omega^{1}, \ldots, \omega^{m}, \partial_{x} \omega^{1}, \ldots, \partial_{x}^{2 j-1} \omega^{m}\right)=0
$$

for $k=1, \ldots, m=m(j)$ where the nonlinear terms, $Q_{k}$, are independent of the highest derivatives, i.e., those of order $2 j$. To study system (1.3) we follow the arguments in [10]. In this case some modifications are needed since the $Q_{k}$ 's introduced by the change of variable involve nonlocal operators.

The use of a change of dependent variable was suggested by recent works of Hayashi and Ozawa [4, 5] on nonlinear Schrödinger equations (see also [13]). In these works this change of variable was called a gauge transformation.

The main results of this paper are the following.

Theorem 1.1. Let $P(\cdot)$ be a polynomial of the type described in (1.2). Then there exist $s, m \in \mathbb{Z}^{+}$such that for any $u_{0} \in H^{s}(\mathbb{R}) \cap L^{2}\left(|x|^{m} d x\right)=X_{s, m}$ there exist $T=T\left(\left\|u_{0}\right\|_{X_{s, m}}\right)>0$ (with $T(\alpha) \rightarrow \infty$ as $\alpha \rightarrow 0$ ) and a unique solution $u(\cdot)$ of the IVP (1.1) satisfying

$$
\begin{gathered}
u \in C\left([0, T]: X_{s, m}\right), \\
\sup _{x} \int_{0}^{T}\left|\partial_{x}^{s+j} u(x, t)\right|^{2} d t<\infty,
\end{gathered}
$$

and

$$
\int_{-\infty[0, T]}^{\infty} \sup _{x}\left|\partial_{x}^{r} u(x, t)\right| d x<\infty, \quad r=0, \ldots,\left[\frac{s+j}{2}\right] .
$$

If $u_{0} \in X_{s_{o}, m}$ with $s_{o}>s$, the results above hold with $s_{o}$ instead of $s$ in the same time interval $[0, T]$.

Moreover, for any $T^{\prime} \in(0, T)$ there exists a neighborhood $U_{u_{0}}$ of $u_{0}$ in $X_{s, m}$ such that the map $\widetilde{u}_{0} \mapsto \widetilde{u}(t)$ from $U_{u_{0}}$ into the class defined in (1.4)-(1.6), with $T^{\prime}$ instead of $T$, is Lipschitz.

Theorem 1.2. Let $P(\cdot)$ be a polynomial of the type described in (1.2) with $\ell_{0} \geq 3$. Then the results in Theorem 1.1 hold with $m=0$ and $L_{x}^{2}$-norm instead of $L_{x}^{1}$-norm in (1.6).

Theorem 1.2 tells us that the IVP for the equation

$$
\partial_{t} u+\partial_{x}^{3} u+\left(u^{2}+\left(\partial_{x} u\right)^{2}\right) \partial_{x}^{2} u=0, \quad t, x \in \mathbb{R},
$$

is locally well posed in $H^{s}(\mathbb{R}), s \geq s_{0}$, with $s_{0}$ sufficiently large. We observe that a similar result does not hold for the parabolic problem

$$
\partial_{t} u-\partial_{x}^{2} u+\left(u^{2}+\left(\partial_{x} u\right)^{2}\right) \partial_{x}^{2} u=0, \quad t>0, x \in \mathbb{R}
$$

Roughly speaking Theorems 1.1-1.2 establish conditions which guarantee that the local behavior of the solution of $(1.1)$ is controlled by the linear part of the 
equation. Moreover, it shows that the dispersive structure of the equation is strong enough to overcome nonlinear terms of lower order with arbitrary sign as in (1.7).

Finally, we observe that Theorems 1.1-1.2 extend to equations of the form

$$
\partial_{t} u+H \partial_{x}^{2 j} u+P\left(u, H u, \ldots, \partial_{x}^{2 j-2} u, H \partial_{x}^{2 j-2} u, \partial_{x}^{2 j-1} u\right)=0
$$

and

$$
\partial_{t} u+\partial_{x}^{2 j+1} u+P\left(u, H u, \ldots, \partial_{x}^{2 j-2} u, H \partial_{x}^{2 j-2} u, H \partial_{x}^{2 j-1} u\right)=0
$$

(where $H$ denotes the Hilbert transform in the space variable and $P(\cdot)$ is as in (1.2)), which generalizes the Benjamin-Ono hierarchy [15], and they extend to systems as

$$
\left\{\begin{array}{c}
\partial_{t} u^{1}+\partial_{x}^{2 j+1} u^{1}+P_{1}\left(u^{1}, \ldots, u^{m}, \ldots, \partial_{x}^{2 j-1} u^{1}, \ldots, \partial_{x}^{2 j-1} u^{m}, \partial_{x}^{2 j} u^{1}\right)=0 \\
\partial_{t} u^{k}+\partial_{x}^{2 j+1} u^{k}+P_{k}\left(u^{1}, \ldots, u^{m}, \ldots, \partial_{x}^{2 j-1} u^{1}, \ldots, \partial_{x}^{2 j-1} u^{m}\right)=0 \\
k=2, \ldots, m
\end{array}\right.
$$

and more generally

$$
\begin{array}{r}
\partial_{t} u^{k}+\partial_{x}^{2 j+1} u^{k}+P_{k}\left(u^{1}, \ldots, u^{m}, \ldots, \partial_{x}^{2 j} u^{1}, \ldots, \partial_{x}^{2 j} u^{m}\right)=0, \\
k=1, \ldots, m,
\end{array}
$$

which contain those deduced in [16] (for example, [16, (1.7)] is a particular case of $(1.8 \mathrm{a})$ and $[16,(1.13),(3.29)]$ belong to the class in $(1.8 \mathrm{~b}))$. In the case (1.8b) our results apply only to small data.

The authors thank J.-C. Saut for pointing out these applications of our results.

The plan of the paper is as follows. In $\S 2$ we state all the linear estimates needed in the proof of Theorems 1.1 and 1.2. In $\S 3$ we explain how to use a change of dependent variable to write the equation in (1.1) as a system in (1.3). To simplify the exposition we shall only consider the case $j=1$. The general case follows the same pattern. Finally in $\S 4$ we prove Theorems 1.1-1.2. Again for simplicity of the exposition we shall take the case $j=1$ and $\ell_{0} \geq 3$ (Theorem 1.2). The former assumption allows us to work in $H^{s}(\mathbb{R})$ and thus to avoid the use of weighted spaces. The techniques needed in the general case $\ell_{0}=2$-weighted norms, the use of the vector fields $x+(2 j+1) t \partial_{x}^{2 j}$, and so on-have been explained in detail in $[8,10]$.

\section{LINEAR ESTIMATES}

We begin with the following sharp homogeneous and inhomogeneous versions of the local smoothing effect of Kato type [6] exhibited by the group $\left\{e^{t \partial_{x}^{2 j}}\right\}_{-\infty}^{\infty}$.

Theorem 2.1. There exist constants, $c_{j}$, such that for each $x \in \mathbb{R}$

$$
\left(\int_{-\infty}^{\infty}\left|\partial_{x}^{j} e^{t \partial_{x}^{2 j+1}} u_{0}(x)\right|^{2} d t\right)^{\frac{1}{2}}=c_{j}\left\|u_{0}\right\|_{2}
$$

There exist constants, $\bar{c}_{j}$, such that

$$
\begin{aligned}
& \left\|\partial_{x}^{2 j} \int_{0}^{t} e^{\left(t-t^{\prime}\right) \partial_{x}^{2 j+1} F\left(\cdot, t^{\prime}\right) d t^{\prime}}\right\|_{L_{x}^{\infty}\left(\mathbf{R}: L_{t}^{2}(\mathbf{R})\right)} \\
& \quad \equiv \sup _{x}\left\|\partial_{x}^{2 j} \int_{0}^{t} e^{\left(t-t^{\prime}\right) \partial_{x}^{2 j+1}} F\left(\cdot, t^{\prime}\right) d t^{\prime}\right\|_{L_{l}^{2}(\mathbf{R})} \leq \bar{c}_{j}\|F\|_{L_{x}^{1}\left(\mathbf{R}: L_{l}^{2}(\mathbf{R})\right)} .
\end{aligned}
$$


Proof. Version (2.1) of the homogeneous local smoothing effect was proven in $[7, \S 4]$ (for previous results in this direction see [1, 20,21]).

(2.2) was first established in [8, Theorem 2.3] (see also [9, §3]). Notice that the gain of derivatives in the inhomogeneous case (2.2) doubles that in (2.1).

Corollary 2.2. For any $T>0$

$$
\left\|\partial_{x}^{j+\ell} \int_{0}^{t} e^{\left(t-t^{\prime}\right) \partial_{x}^{2 j+1}} F\left(\cdot, t^{\prime}\right) d t^{\prime}\right\|_{L_{x}^{\infty}\left(\mathbf{R}: L_{t}^{2}([0, T])\right)} \leq c T^{\alpha}\|F\|_{L_{x}^{p}\left(\mathbf{R}: L_{t}^{2}([0, T])\right)}
$$

with $\ell=0,1, \ldots, j, \alpha=(j-\ell) / 2 j$, and $p=2 j /(j+\ell)$.

Proof. Combining (2.1), Minkowski's integral inequality, and the CauchySchwarz inequality one finds that

$$
\begin{aligned}
& \left\|\partial_{x}^{j} \int_{0}^{t} e^{\left(t-t^{\prime}\right) \partial_{x}^{2 j+1} F\left(\cdot, t^{\prime}\right) d t^{\prime}}\right\|_{L_{x}^{\infty}\left(\mathbb{R}: L_{t}^{2}([0, T])\right)} \\
& \quad \leq c \int_{0}^{T}\|F(\cdot, t)\|_{2} d t \leq c T^{\frac{1}{2}}\|F\|_{L_{x}^{2}\left(\mathbb{R}: L_{t}^{2}([0, T])\right)} .
\end{aligned}
$$

Hence (2.3) follows by interpolation between (2.2) and (2.4).

As in $[8,9]$ to complement the above estimate we need to bound the $L^{p}$-norm of the maximal function $\sup _{[0, T]}\left|e^{t t_{x}^{2 j+1}} u_{0}(x)\right|$. For our purpose here it suffices to consider the case $p=2$.

Theorem 2.3. For any $\rho>3 / 4$ and any $s>(2 j+1) / 4$ there exists $c$ such that

$$
\begin{aligned}
\left\|e^{t t_{x}^{2 j+1}} u_{0}\right\|_{L_{x}^{2}\left(\mathbf{R}: L_{t}^{\infty}([0, T])\right)} & \equiv\left(\int_{-\infty[0, T]}^{\infty} \sup \left|e^{t \partial_{x}^{2 j+1}} u_{0}(x)\right|^{2} d x\right)^{\frac{1}{2}} \\
& \leq c(1+T)^{\rho}\left\|u_{0}\right\|_{H^{s}} .
\end{aligned}
$$

Proof. See [8, 9].

We observe that a global (in time) version of (2.5) can be obtained only when $L_{x}^{2}$ is replaced by $L_{x}^{p}$ with $p \geq 4$ (see [11]). On the other hand to estimate the $L_{x}^{1}$-norm of the maximal function (as in (1.6)) one has to use weighted norms, i.e., $L^{2}\left(|x|^{m} d x\right)$-norm. These are essential in the proof of Theorem 1.1. Here we shall restrict ourselves to the case $\ell_{0} \geq 3$ in (1.2) (Theorem 1.2), for which estimate (2.5) suffices.

\section{A CHANGE OF DEPENDENT VARIABLE}

In this section we shall show how to use a gauge transformation to reduce the equation in (1.1) to a system of the type (1.3). The advantage is that the nonlinear terms in (1.3) do not involve the highest derivatives, i.e., those of order $2 j$. As was mentioned above the use of a gauge transformation was suggested by recent works of Hayashi and Ozawa $[4,5]$. To simplify the exposition we shall restrict ourselves to the case $j=1$; i.e.,

$$
\partial_{t} u+\partial_{x}^{3} u+P\left(u, \partial_{x} u, \partial_{x}^{2} u\right)=0 .
$$


Differentiating the equation (3.1) six times (for technical convenience) and introducing the variables

$$
\left\{\begin{array}{l}
\omega_{k}=\partial_{x}^{k} u \quad \text { for } k=0,1, \ldots, 5, \\
\omega_{6}=\partial_{x}^{6} u \Phi \text { with } \Phi=\Phi(x, t) \text { to be determined }
\end{array}\right.
$$

after some straightforward computations we find that

$$
\begin{aligned}
\partial_{t} \omega_{k}+\partial_{x}^{3} \omega_{k} & +P_{k}\left(\omega_{0}, \ldots, \omega_{k+1}, \partial_{x} \omega_{k+1}\right)=0, \quad k=0,1, \ldots, 4 \\
\partial_{t} \omega_{5} & +\partial_{x}^{3} \omega_{5}+\widetilde{P}_{5}\left(\omega_{0}, \ldots, \omega_{5}, \omega_{6} \Phi^{-1}\right) \\
& +\frac{\partial P}{\partial \omega_{2}}\left(\omega_{0}, \omega_{1}, \omega_{2}\right)\left(\partial_{x} \omega_{6} \Phi^{-1}-\omega_{6} \Phi^{-2} \partial_{x} \Phi\right)
\end{aligned}
$$

where $P_{0}, \ldots, P_{4}, \widetilde{P}_{5}$ are polynomials in the class (1.2) with the same $\ell_{0}$ as $P$, and

$$
\begin{gathered}
\partial_{t} \omega_{6}+\partial_{x}^{3} \omega_{6}-\partial_{t} \Phi \Phi^{-1} \omega_{6}-3 \partial_{x} \Phi \partial_{x}^{8} u-3 \partial_{x}^{2} \Phi \partial_{x}\left(\omega_{6} \Phi^{-1}\right)-\partial_{x}^{3} \Phi\left(\omega_{6} \Phi^{-1}\right) \\
+\frac{\partial P}{\partial \omega_{2}}\left(\omega_{0}, \omega_{1}, \omega_{2}\right) \Phi \partial_{x}^{8} u+\widetilde{P}_{6}\left(\Phi, \Phi^{-1}, \omega_{0}, \ldots, \omega_{6}, \partial_{x} \omega_{6}\right)=0
\end{gathered}
$$

We must choose $\Phi$ such that the two terms in (3.5) involving $\partial_{x}^{8} u$ cancel. Hence

$$
-3 \partial_{x} \Phi+\frac{\partial P}{\partial \omega_{2}}\left(\omega_{0}, \omega_{1}, \omega_{2}\right) \Phi=0
$$

i.e.,

$$
\Phi(x, t)=\exp \left(\frac{1}{3} \int_{-\infty}^{x} \frac{\partial P}{\partial \omega_{2}}\left(\omega_{0}, \omega_{1}, \omega_{2}\right)\left(x^{\prime}, t\right) d x^{\prime}\right) .
$$

Now using (3.1) one sees that

$$
\begin{aligned}
\partial_{t} \boldsymbol{\Phi} & =\frac{1}{3} \boldsymbol{\Phi}\left(\int_{-\infty}^{x} \frac{\partial}{\partial t}\left(\frac{\partial}{\partial \omega_{2}} P\left(\omega_{0}, \omega_{1}, \omega_{2}\right)\left(x^{\prime}, t\right)\right) d x^{\prime}\right) \\
& =\boldsymbol{\Phi}\left(\int_{-\infty}^{x} \widetilde{P}\left(\omega_{0}, \ldots, \omega_{5}\right)\left(x^{\prime}, t\right) d x^{\prime}\right)
\end{aligned}
$$

where $\widetilde{P}$ is a polynomials in the class (1.2) with $\ell_{0}-1$ instead of $\ell_{0}$. Thus when $\ell_{0}=2$ one has to use the differential equation and the weighted norm $\left(L^{2}\left(|x|^{m} d x\right)\right)$ to guarantee that $\widetilde{P}(\cdot, t) \in L^{1}(\mathbb{R})$.

Combining (3.6) and (3.7) we can rewrite (3.5)

$$
\begin{aligned}
\partial_{t} \omega_{6} & +\partial_{x}^{3} \omega_{6}-\left(\int_{-\infty}^{x} \widetilde{P}\left(\omega_{0}, \ldots, \omega_{5}\right)\left(x^{\prime}, t\right) d x^{\prime}\right) \omega_{6} \\
& +P_{6}\left(\Phi, \omega_{0}, \ldots, \omega_{6}, \partial_{x} \omega_{6}\right)=0
\end{aligned}
$$


with $P_{6}\left(\Phi, \omega_{0}, \ldots, \omega_{6}, \partial_{x} \omega_{6}\right)$ satisfying that $P_{6}\left(y, z_{0}, \ldots, z_{6}, z_{7}\right)$ is a polynomial in (1.2) for a fixed $y$, with the same $\ell_{0}$ as $P(\cdot)$ in (3.1). Thus the system (3.3), (3.8) is of the form described in (1.3).

\section{Proof of Theorem 1.2}

In the previous section we showed that the IVP (1.1) can be written as (4.1)

$$
\left\{\begin{array}{l}
\partial_{t} \omega_{k}+\partial_{x}^{3} \omega_{k}+P_{k}\left(\omega_{0}, \ldots, \omega_{k+1}, \partial_{x} \omega_{k+1}\right)=0, \quad k=0,1, \ldots, 4, \\
\partial_{t} \omega_{5}+\partial_{x}^{3} \omega_{5}+P_{5}\left(\Phi, \omega_{0}, \ldots, \omega_{6}, \partial_{x} \omega_{6}\right)=0, \\
\partial_{t} \omega_{6}+\partial_{x}^{3} \omega_{6}-\left(\int_{-\infty}^{x} \widetilde{P}\left(\omega_{0}, \ldots, \omega_{5}\right)\left(x^{\prime}, t\right) d x^{\prime}\right) \omega_{6} \\
\quad+P_{6}\left(\Phi, \omega_{0}, \ldots, \omega_{6}, \partial_{x} \omega_{6}\right)=0, \\
\left(\omega_{0}, \ldots, \omega_{6}\right)(x, 0)=\left(u_{0}(x), \ldots, \partial_{x}^{5} u_{0}(x), \partial_{x}^{6} u_{0}(x) \Phi(x, 0)\right)
\end{array}\right.
$$

with $\Phi=\Phi(\omega)$ as in (3.6) and where, for a fixed $z, P_{0}(\cdot), \ldots, P_{4}(\cdot), P_{5}(z, \cdot)$, $P_{6}(z, \cdot)$ are polynomials in the class (1.2) with the same $\ell_{0}$ as $P$ in (1.1), and the same holds for $\widetilde{P}(\cdot)$ with $\ell_{0}-1$ instead of $\ell_{0}$.

As was mentioned in the introduction we shall consider only the case $k \geq 3$ (Theorem 1.2). In this situation the data $u_{0} \in H^{s}(\mathbb{R})$. Thus $\omega(0)=$ $\left(\omega_{0}, \ldots, \omega_{6}\right)(x, 0) \in\left(H^{s^{\prime}}(\mathbb{R})\right)^{7}$ with $s^{\prime}=s-6$ to be determined.

In the proof of Theorem 1.2 we shall use the following notation:

$$
\omega(t)=\left(\omega_{0}, \ldots, \omega_{6}\right)(\cdot, t)
$$

for any $T>0$,

$$
\begin{gathered}
\Gamma_{1}^{T}(\omega)=\max _{k=0, \ldots, 6[0, T]} \sup _{[0, T]}\left\|\omega_{k}(t)\right\|_{H^{s^{\prime}}}, \\
\Gamma_{2}^{T}=\sup _{x}\left(\int_{0}^{T}\left|\partial_{x}^{s^{\prime}+1} \omega_{6}(x, t)\right|^{2} d t\right)^{\frac{1}{2}}, \\
\Gamma_{3}^{T}=\max _{k=0, \ldots, 6} \sup _{n=0, \ldots, r}\left(\int_{-\infty[0, T]}^{\infty} \sup _{x}\left|\partial_{x}^{n} \omega_{k}(x, t)\right|^{2} d x\right)^{\frac{1}{2}}
\end{gathered}
$$

with $r=\left[s^{\prime} / 2\right],[\cdot]$ the greatest integer function,

$$
\Omega^{T}(\omega)=\max \left\{\Gamma_{\beta}^{T}(\omega) / \beta=1,2,3\right\},
$$

and

$$
Y_{T}^{a}=\left\{\omega: \mathbb{C} \times[0, T] \rightarrow \mathbb{C}^{7} / \Omega^{T}(\omega) \leq a\right\}
$$

Writing the system (4.1) in the integral form

$$
\omega(t)=\omega(0)+\int_{0}^{t} e^{\left(t-t^{\prime}\right) \partial_{x}^{3}} \mathscr{P}\left(\omega, \partial_{x} \omega\right)\left(t^{\prime}\right) d t^{\prime}
$$

with $\mathscr{P}=\left(-P_{1}, \ldots,-P_{5},\left(\int_{-\infty}^{x} \widetilde{P}\left(\omega_{0}, \ldots, \omega_{5}\right)\left(x^{\prime}, t\right) d x^{\prime}\right) \omega_{6}-P_{6}\right)$ we reduce the problem to showing that the operator

$$
\Lambda(v)(t)=\Lambda_{\omega(0)}(v)(t)=\omega(0)+\int_{0}^{t} e^{\left(t-t^{\prime}\right) \partial_{x}^{3}} \mathscr{P}\left(v, \partial_{x} v\right)\left(t^{\prime}\right) d t^{\prime}
$$


defines a contraction in $Y_{T}^{a}$ for appropriate values of $a, T$.

From our assumption on $P(\cdot)$ it is easy to verify that the nonlinear terms of lowest order in $\mathscr{P}(\cdot)(4.7)$ (see (3.3)-(3.8)) have one of the following forms for $i, k, m \in\{0, \ldots, 5\}$ :

$$
\omega_{i} \omega_{k} \omega_{m}
$$

$$
\omega_{i} \omega_{k} \omega_{6} \Phi^{-1}
$$

or

$$
\left(\int_{-\infty}^{x} \widetilde{P}\left(\omega_{0}, \ldots, \omega_{5}\right)\left(x^{\prime}, t\right) d x^{\prime}\right) \omega_{6} .
$$

In order to prove Theorem 1.2 we need the following estimates.

Proposition 4.1. For $i, k, m \in\{1, \ldots, 5\}$ and $s^{\prime} \geq 2$

$$
\int_{0}^{T}\left\|\omega_{i} \omega_{k} \omega_{m}(t)\right\|_{H^{\prime}} d t \leq c T\left(\Gamma_{1}^{T}(\omega)\right)^{3},
$$

$$
\begin{aligned}
& \int_{0}^{T}\left\|\omega_{i} \omega_{k} \omega_{6} \Phi^{-1}(t)\right\|_{H^{s^{\prime}}} d t \\
& \quad \leq c T\left(\left(\Gamma_{1}^{T}(\omega)\right)^{3}+\left(\Gamma_{1}^{T}(\omega)\right)^{s^{\prime} \ell_{1}}\right) \exp \left(\left(\Gamma_{1}^{T}(\omega)\right)^{2}+\left(\Gamma_{1}^{T}(\omega)\right)^{\ell_{1}-1}\right) \equiv D_{1}
\end{aligned}
$$

with $\ell$ as in (1.2),

$$
\begin{aligned}
& \int_{0}^{T}\left\|\omega_{i} \omega_{k} \partial_{x} \omega_{6} \Phi^{-1}(t)\right\|_{H^{s^{\prime}}} d t \\
& \quad \leq D_{1}+c T^{\frac{1}{2}} \Gamma_{1}^{T}(\omega) \Gamma_{2}^{T}(\omega) \Gamma_{3}^{T}(\omega) \exp \left(\left(\Gamma_{1}^{T}(\omega)\right)^{2}+\left(\Gamma_{1}^{T}(\omega)\right)^{\ell_{1}-1}\right)
\end{aligned}
$$

and

$$
\begin{aligned}
\int_{0}^{T} & \left\|\left(\int_{-\infty}^{x} \tilde{P}\left(\omega_{0}, \ldots, \omega_{5}\right)\left(x^{\prime}, t\right) d x^{\prime}\right) \omega_{6}(t)\right\|_{H^{s^{\prime}}} d t \\
& \leq c T\left(\left(\Gamma_{1}^{T}(\omega)\right)^{3}+\left(\Gamma_{1}^{T}(\omega)\right)^{s^{\prime} \ell_{1}}\right) .
\end{aligned}
$$

Proof. Estimate (4.9) follows by combining the Leibniz rule and the GagliardoNirenberg inequality.

To obtain (4.10) one needs (4.9) and the estimate

$$
\begin{aligned}
\sup _{[0, T]}\left\|\Phi^{-1}(\cdot, t)\right\|_{\infty} & \leq c \exp \left(\sup _{[0, T]}\left\|\frac{\partial P}{\partial \omega_{2}}\left(\omega_{0}, \omega_{1}, \omega_{2}\right)(t)\right\|_{1}\right) \\
& \leq \exp \left(\left(\Gamma_{1}^{T}(\omega)\right)^{2}+\left(\Gamma_{1}^{T}(\omega)^{\ell_{1}-1}\right)\right) \equiv D_{2}
\end{aligned}
$$

$(\operatorname{see}(3.6))$. 
Estimate (4.11) follows from (4.10), the Leibniz rule, and the inequality (4.14)

$$
\begin{aligned}
\int_{0}^{T} & \left\|\omega_{i} \omega_{k} \partial_{x}^{s^{\prime}+1} \omega_{6} \Phi^{-1}(t)\right\|_{2} d t \\
& \leq\left(\int_{0}^{T}\left\|\omega_{i}(t)\right\|_{\infty}^{2} d t\right)^{\frac{1}{2}}\left(\int_{0}^{T}\left\|\omega_{k} \partial_{x}^{s^{\prime}+1} \omega_{6}(t)\right\|_{2}^{2} d t\right)^{\frac{1}{2}} \sup _{[0, T]}\left\|\Phi^{-1}(t)\right\|_{\infty} \\
& \leq c T^{\frac{1}{2}} \Gamma_{1}^{T}(\omega)\left\|\omega_{k}\right\|_{L_{x}^{2}\left(\mathbf{R}: L_{t}^{\infty}([0, T])\right)}\left\|\partial_{x}^{s^{\prime}+1} \omega_{6}\right\|_{L_{x}^{\infty}\left(\mathbf{R}: L_{t}^{2}([0, T])\right)} D_{2} \\
& \leq c T^{\frac{1}{2}} \Gamma_{1}^{T}(\omega) \Gamma_{3}^{T}(\omega) \Gamma_{2}^{T}(\omega) D_{2} .
\end{aligned}
$$

In (4.14) we used (4.13), Holder's inequality, and Sobolev's theorem.

Finally (4.12) follows by combining the Leibniz rule and the inequality

$$
\begin{aligned}
\sup _{[0, T]} & \left\|\left(\int_{-\infty}^{x} \widetilde{P}\left(\omega_{0}, \ldots, \omega_{5}\right)\left(x^{\prime}, t\right) d x^{\prime}\right) \partial_{x}^{s^{\prime}} \omega_{6}\right\|_{2} \\
& \leq \sup _{[0, T]}\left\|\widetilde{P}\left(\omega_{0}, \ldots, \omega_{5}\right)(t)\right\|_{1} \Gamma_{1}^{T}(\omega) \leq c\left(\left(\Gamma_{1}^{T}(\omega)\right)^{3}+\left(\Gamma_{1}^{T}(\omega)\right)_{1}^{\ell_{1}}\right) .
\end{aligned}
$$

We observe that the corresponding estimates for the nonlinear terms in $\mathscr{P}(\cdot)$ (4.7) of higher order, i.e., $4, \ldots, \ell_{1}$, follow from a similar argument. Having proved the necessary estimates (Proposition 4.1) we can now turn to the proof of Theorem 1.2.

Combining the group properties of the associated linear problem, (4.9)-(4.12) and the integral equation (4.7), one sees that

$$
\begin{aligned}
\Gamma_{1}^{T}(\Lambda(v)) \leq & c\|\omega(0)\|_{H^{s}}+c T\left(\left(\Gamma_{1}^{T}(\omega)\right)^{3}\right. \\
& \left.+\left(\Gamma_{1}^{T}(\omega)\right)^{\left(s^{\prime}+1\right) \ell_{1}}\right) \exp \left(\left(\Gamma_{1}^{T}(\omega)\right)^{2}+\left(\Gamma_{1}^{T}(\omega)\right)^{\ell_{1}-1}\right) \\
& +c T^{\frac{1}{2}}\left(\Gamma_{1}^{T}(\omega)+\left(\Gamma_{1}^{T}(\omega)\right)^{\ell_{1}-2}\right) \\
& \times \Gamma_{2}^{T}(\omega) \Gamma_{3}^{T}(\omega) \exp \left(\left(\Gamma_{1}^{T}(\omega)\right)^{2}+\left(\Gamma_{1}^{T}(\omega)\right)^{\ell_{1}-1}\right) \equiv D_{3} .
\end{aligned}
$$

Similarly, inserting (2.1) with $j=1$ in (4.7) one has that

$$
\Gamma_{2}^{T}(\Lambda(v)) \leq D_{3} .
$$

Finally assuming $s^{\prime} \geq 4$ from (2.5) it follows that

$$
\Gamma_{3}^{T}(\Lambda(v)) \leq D_{3} .
$$

Collecting (4.15)-(4.17) we find that

$$
\begin{aligned}
\Omega^{T}(\Lambda(v)) \leq & c\|\omega(0)\|_{H^{s^{\prime}}}+c T^{\frac{1}{2}}\left(1+T^{\frac{1}{2}}\right)\left(\left(\Omega^{T}(v)\right)^{3}\right. \\
& \left.+\left(\Omega^{T}(v)\right)^{\left(s^{\prime}+1\right) \ell_{1}}\right) \exp \left(\left(\Omega^{T}(v)\right)^{2}+\left(\Omega^{T}(v)\right)^{\ell_{1}-1}\right) .
\end{aligned}
$$

The above argument combined with the mean value theorem and the form of $\Phi$ in (3.6) shows that

(4.19)

$$
\begin{aligned}
\Omega^{T}(\Lambda(v-\tilde{v})) \leq & c T^{\frac{1}{2}} \Omega^{T}(v-\tilde{v})\left(\left(\Omega^{T}(v)\right)^{2}+\left(\Omega^{T}(\tilde{v})\right)^{2}\right) \\
& \times\left(\left(D_{5}(v)\right)^{s^{\prime} \ell_{1}}+\left(D_{5}(\tilde{v})\right)^{s^{\prime} \ell_{1}}\right) \\
& \times \exp \left(\left(\Omega^{T}(v)\right)^{2}+\left(\Omega^{T}(\tilde{v})\right)^{2}+\left(\Omega^{T}(v)\right)^{\ell_{1}-1}+\left(\Omega^{T}(\tilde{v})\right)^{\ell_{1}-1}\right) .
\end{aligned}
$$


Hence choosing

$$
a=2 c\|\omega(0)\|_{H^{s^{\prime}}}
$$

and $T$ such that

$$
\text { c } T^{\frac{1}{2}}\left(1+T^{\frac{1}{2}}\right)\left(a+a^{s^{\prime} \ell_{1}}\right) \exp \left(a^{2}+a^{\ell_{1}-1}\right) \leq \frac{1}{2}
$$

we obtain that the map $\Lambda(\cdot)$ defined in (4.7) defines a contraction in $Y_{T}^{a}$. This basically completes the proof of Theorem 2.1 (for more details see [8-10]).

\section{Note ADded in Proof}

After this work was completed, Professor W. Craig informed us that his student H. S. Cai generalized some of the results proved here.

\section{REFERENCES}

1. P. Constantin and J.-C. Saut, Local smoothing properties of dispersive equations, J. Amer. Math. Soc. 1 (1988), 413-446.

2. W. Craig, T. Kappeler, and W. A. Strauss, Gain of regularity for equations of KdV type, Ann. Inst. H. Poincaré Anal. Non Linéaire 9 (1992), 147-186.

3. A. S. Fokas, Aspects of integrability in one and several dimensions, Topics in Soliton Theory and Exactly Solvable Nonlinear Equations (M. Ablowitz et al., eds.), World Scientific, Singapore, 1987, pp. 46-77.

4. N. Hayashi and T. Ozawa, On the derivative nonlinear Schrödinger equation, Phys. D 55 (1992), 14-36.

5. __ Remarks on nonlinear Schrödinger equations in one space dimension, Differential Integral Equations (to appear).

6. T. Kato, On the Cauchy problem for the (generalized) Korteweg-de Vries equation, Adv. Math. Suppl. Stud., Stud. Appl. Math. 8 (1983), 93-128.

7. C. E. Kenig, G. Ponce, and L. Vega, Oscillatory integrals and regularity of dispersive equations, Indiana Univ. Math. J. 40 (1991), 33-69.

8. __ Small solutions to nonlinear Schrödinger equation, Ann. Inst. H. Poincaré Anal. Non Linéaire 10 (1993), 255-288.

9. __ Well-posedness and scattering results for the generalized Korteweg-de Vries equation via contraction principle, Comm. Pure Appl. Math. 46 (1993), 527-620.

10. _ On the hierarchy of the generalized KdV equations, Proc. Lyon Workshop on Singular Limits of Dispersive Waves (to appear).

11. C. E. Kenig and A. Ruiz, A strong type $(2,2)$ estimate for the maximal function associated to the Schrödinger equation, Trans. Amer. Math. Soc. 230 (1983), 239-246.

12. S. Kichenassamy and P. J. Olver, Existence and non-existence of solitary waves solutions to higher order model evolution equations, Siam J. Math. Anal. 23 (1992), 1141-1166.

13. A. Kundu, Landau-Lifshitz and higher-order nonlinear systems gauge generated from nonlinearSchrödinger type equations, J. Math. Phys. 25 (1984), 3433-3437.

14. P. D. Lax, Integrals of nonlinear equations of evolution and solitary waves, Comm. Pure Appl. Math. 21 (1965), 467-490.

15. Y. Matsuno, Bilinear Transformation Method, Academic Press, New York, 1984.

16. Y. W. Oevel and Z. Popowicz, The bi-Hamiltonian structure of fully supersymmetric Korteweg-de Vries systems, Comm. Math. Phys. 139 (1991), 441-460.

17. G. Ponce, Lax pairs and higher order models for water waves, J. Differential Equations 102 (1993), 360-381.

18. J.-C. Saut, Sur quelques gènéralisations de l'èquations de Korteweg-de Vries, II, J. Differential Equations 33 (1979), 320-335. 
19. M. Schwarz Jr., The initial value problem for the sequence of generalized Korteweg-de Vries equations, Adv. Math. 54 (1984), 22-56.

20. P. Sjölin, Regularity of solutions to the Schrödinger equation, Duke Math. J. 55 (1987), 699-715.

21. L. Vega, The Schrödinger equations: pointwise convergence to the initial data, Proc. Amer. Math. Soc. 102 (1988), 874-878.

Department of Mathematics, University of Chicago, Chicago, Illinois 60637

Department of Mathematics, University of California, Santa Barbara, California 93106

Facultad De Ciencias, Universidad Autonoma de Madrid, Cantoblanco, Madrid 28049, SPAIN 